\title{
Mesh network dynamic routing protocols
}

\begin{abstract}
The main course of this paper will be to assess the applicability of dynamic routing protocols in Mesh networks. In the process, evaluation criteria were identified, an evaluation method was developed and implemented using simulation in the NS-3 environment, a method for describing a topology has been proposed, and an experiment was conducted using the method on a simulated network topology. The experiment showed the possibility of applying the method to evaluate such indicators as throughput and error rate, loss coefficient, delay variation and network delay. The results obtained allowed to conclude about the applicability of particular routing protocol to specified topology with specific network quality requirements.
\end{abstract}

Keyword: Routing protocols; Mesh; Manet; WMN 